Working Papers

Institute of

Mathematical

Economics

June 2005

Two support results for the Kalai-Smorodinsky solution in small object division markets

Claus-Jochen Haake

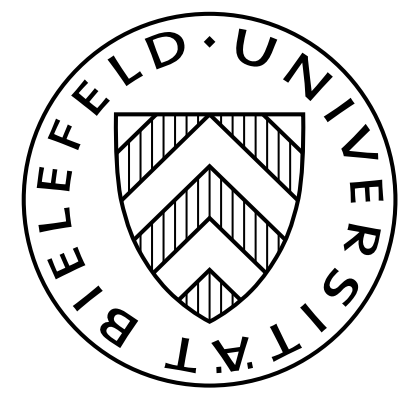




\title{
Two support results for the Kalai-Smorodinsky solution in small object division markets
}

\author{
Claus-Jochen Haake ${ }^{1}$ \\ Institute of Mathematical Economics (IMW). \\ Bielefeld University \\ CHaake@wiwi.uni-bielefeld.de
}

June 8, 2005

\begin{abstract}
We discuss two support results for the Kalai-Smorodinsky bargaining solution in the context of an object division problem involving two agents. Allocations of objects resulting from strategic interaction are obtained as a demand vector in a specific market. For the first support result games in strategic form are derived that exhibit a unique Nash equilibrium. The second result uses subgame perfect equilibria of a game in extensive form. Although there may be multiple equilibria, coordination problems can be removed.
\end{abstract}

Keywords: support result, object division, market, Kalai-Smorodinsky solution JEL Classification: C78, D40

\section{Introduction}

The task to obtain a non-cooperative foundation for a cooperative solution concept is widely described by the term Nash program. Starting with an underlying cooperative game, one needs to derive a non-cooperative game in normal or extensive form in such a way that equilibrium payoffs coincide with (or belong to) the cooperative solution. In this paper, we want to support the Kalai-Smorodinsky (hereafter KS) bargaining solution ${ }^{2}$ in this spirit. In effect, the bargaining solution can as well be obtained by strategic interaction instead of signing an agreement. Clearly, one is interested in formulating a universal way to derive the non-cooperative game, which means that one should not make use of particular knowledge of the underlying bargaining problem.

\footnotetext{
${ }^{1}$ I thank Walter Trockel for valuable comments. Financial support from the project "Fairness und Anreize", FiF-Projekt, Bielefeld University is gratefully acknowledged.

${ }^{2}$ see Kalai \& Smorodinsky (1975).
} 
Apparently, there are striking similarities between the Nash program and the theory of implementation, although the foci are slightly different. However, Trockel (2002) shows that for solutions concepts from cooperative game theory, a support result as discussed above can be "transformed" into an implementation result in the spirit of implementation theory (see also Serrano (1997), Dagan \& Serrano (1998) and Bergin \& Duggan (1999)). This is the content of the so-called embedding lemma. In particular, any support result for a bargaining solution readily yields an implementation result for this solution as well.

Roughly, there are two ways, in which we could think about supporting a bargaining solution. One way is to follow a purely welfaristic approach, which means that one only considers the possible allocations of utility provided in the bargaining situation and takes this set (together with the status quo point) as the primitives of the cooperative model. Along this line, Trockel (1999) discusses support results for a class of bargaining solutions including the KS solution (cf. also Haake (2000)).

In the second direction, there is an additional entity considered in the model; a set of outcomes. This set may, for example, consist of allocations of goods or (lotteries over) alternatives. A bargaining problem is induced by evaluation of outcomes with individual utility functions. Therefore, supporting a bargaining solution in such a setup means achieving a certain outcome through strategic interaction, the utility of which coincides with the given bargaining solution. Such a non-welfaristic approach can cure an important drawback: In practice it is not necessarily clear, how a specific utility allocation is actually obtained, whereas it appears to be a much simpler task to select a certain (physical) outcome. In a seminal paper, Moulin (1984) discusses an implementation of the KS solution, using a mechanism, in which "fractions of dictatorship are auctioned off". The winning bid in the auction later constitutes a probability distribution on the set of (physical) alternatives, so that the KS solution is the expected payoff from this lottery. In a similar spirit, Miyagawa (2002) obtains a subgame-perfect implementation result for a class of bargaining solutions including the KS solution.

As Moulin's (1984) work does, we follow the second approach. We investigate an object division problem, in which a finite number of (divisible) objects may be distributed among two agents. Our approach differs from Moulin's in two respects. First, we obtain an exact support result in the sense that deterministic payoffs in equilibrium coincide with the KS solution. And second, instead of using an auction mechanism, we set up an exchange market. Payoffs (in equilibrium) are the result of individual demand. Roughly speaking, a strategy choice in the supporting games determines prices and income, that in turn yield utility maximizing allocations of objects.

We present two support results in the paper: First, we derive from any object division problem a non-cooperative game in strategic form, which has a unique Nash equilibrium. This game gives rise to a second game in extensive form. There the payoff in any subgame perfect equilibrium coincides with the KS solution. Although we cannot guarantee uniqueness of equilibria, no coordination problem occurs, as the resulting outcomes are (essentially) unique. 


\section{Notation and Framework}

We investigate a market, in which finitely many objects are distributed among two agents. Let $I=\{1,2\}$ denote the set of agents and $N:=\{1, \ldots, n\}$ the set of objects. There is exactly one unit of each object in the market. Denote by $e:=(1, \ldots, 1) \in \mathbb{R}^{n}$ the vector of total endowments. We assume that objects are divisible, so that an allocation (of objects) is described by a pair $x=\left(x^{1}, x^{2}\right) \in[0,1]^{n} \times[0,1]^{n}$ satisfying $x^{1}+x^{2} \leq e$. We denote the set of allocations by $\mathcal{A}$. Neither agent is initially endowed with some object. We assume that agents' preferences are linear over divisions of an object and additively separable across objects. That means, agent $i$ 's preferences may be represented by a vector $u^{i}=\left(u_{1}^{i}, \ldots, u_{n}^{i}\right) \gg 0 .^{3}$ The quantity $u_{j}^{i}$ may be interpreted as agent $i$ 's willingness to pay for object $j$. Agent $i$ 's utilities over bundles are given by a function $U^{i}:[0,1]^{n} \longrightarrow \mathbb{R}$, defined by $U^{i}\left(x^{i}\right):=\sum_{j \in N} x_{j}^{i} \cdot u_{j}^{i}(i \in I)$. By $\mathcal{U}$, we denote the set of all pairs of utility functions $\left(U^{1}, U^{2}\right)$ as above. For presentational reasons we will assume that for each pair in $\mathcal{U}$ the corresponding utility vectors $u^{1}, u^{2}$ satisfy $u_{j}^{1} / u_{j}^{2}>u_{j+1}^{1} / u_{j+1}^{2}$ for all $j=1, \ldots, n-1$, which means in particular that no two objects exhibit the same rate of substitution between the two agents. To sum, we describe a market by a tuple $\mathcal{M}=(I, N, U)$ with $U \in \mathcal{U}$.

In the sequel, we want to achieve allocations by letting agents demand objects according to specific income and prices. A price system is a vector $p \in \mathbb{R}_{+}^{n}$. For given price system $p$ and income level $m \in \mathbb{R}_{+}$, we define the budget set $B(m, p):=\left\{y \in[0,1]^{n} \mid p y \leq m\right\}$ and for $i \in I$ agent $i$ 's demand correspondence $D^{i}(m, p):=\operatorname{argmax}\left\{U^{i}(y) \mid y \in B(m, p)\right\}$. In the remainder, we make use of two specific price systems. Due to our assumption that utility functions are representable by a vector $u^{i} \in \mathbb{R}_{++}^{n}$, we may also view $u^{i}$ as a linear function to evaluate (bundles of) objects; hence, we may view $u^{i}$ as a specific price system. Throughout the paper, we set $\bar{p}^{i}:=u^{i}(i \in I)$.

Any market $(I, N, U)$ induces a two person bargaining game in the following way. The set of feasible utility allocations is given by $V^{U}:=\operatorname{compH}\left(\left\{U^{1}\left(y^{1}\right), U^{2}\left(y^{2}\right) \in \mathbb{R}^{2} \mid y \in \mathcal{A}\right\}\right){ }^{4}$ Status quo utilities are (always) given by the origin $0 \in \mathbb{R}^{2}$ and so we identify the game with its utility possibility set $V^{U}$. It is easy to see that $V^{U}$ can be written as a sum of utility possibility sets; one for each object separately, i.e. $V^{U}=\sum_{j \in N} V_{j}^{U}$ with $V_{j}^{U}:=\operatorname{compH}\left(\left\{\lambda\left(u_{j}^{1}, 0\right)+(1-\lambda)\left(0, u_{j}^{2}\right) \mid 0 \leq \lambda \leq 1\right\}\right)$. Hence, the class $\mathcal{V}:=\left\{V^{U} \mid U \in \mathcal{U}\right\}$ of bargaining games generated by an object division problem is the class of games with compactly generated, polyhedral utility possibility sets. For $U \in \mathcal{U}$ define $M^{i}=M^{i, U^{i}}:=U^{i}(e)$. $M^{i}$ reflects agent $i$ 's maximal possible utility in $V^{U}$. The Kalai-Smorodinsky bargaining solution is the mapping $K: \mathcal{V} \longrightarrow \mathbb{R}^{2}$ that takes each $V^{U}$ to its unique Pareto optimal utility allocation, in which both agents obtain the same fraction of their maximal utility, i.e. $K^{1}\left(V^{U}\right) / M^{1, U}=K^{2}\left(V^{U}\right) / M^{2, U}=: \tau\left(V^{U}\right)$.

We close with some important observation on the demand correspondence. Define a parametrization $h=h^{U}:[0,1] \longrightarrow \mathbb{R}^{2}$ of the Pareto boundary of $V^{U}$ through $h(\delta):=$ $\left(\bar{t}(\delta), \delta \cdot M^{2}\right)$, with $\bar{t}(\delta):=\max \left\{t \in R \mid\left(t, \delta \cdot M^{2}\right) \in V^{U}\right\}$. That means, to each $\delta \in[0,1]$ the point $h(\delta)$ is the unique Pareto efficient point in $V^{U}$, in which agent 2 receives a $\delta$ share of his maximal utility. As one easily sees, $h_{1}^{U}$ is a concave, strictly decreasing function. In the

\footnotetext{
${ }^{3}$ We use the following notation for vector inequalities in $\mathbb{R}^{n}: x \gg y$ means $x_{j}>y_{j}(j \in N) ; x>y$ means $x_{j} \geq y_{j}(j \in N)$ and $x \neq y ; x \geq y$ means $x>y$ or $x=y$.

${ }^{4}$ Here $\operatorname{compH}(\cdot)$ denotes the comprehensive hull operator.
} 
following lemma we show that certain demand sets $D^{i}(m, p)$ are singletons. In such cases we will identify the set with its single element.

Lemma 1. Let $\mathcal{M}=(I, N, U)$ be a market satisfying the above assumptions.

1. For $i \in I$ and $m \in\left[0, M^{3-i}\right]$, the demand set $D^{i}\left(m, \bar{p}^{3-i}\right)$ is a singleton.

2. To each Pareto optimal utility allocation $\left(v^{1}, v^{2}\right) \in V^{U}$ there exists a unique (Pareto optimal) allocation $\left(z^{1}, z^{2}\right) \in \mathcal{A}$ with $U^{i}\left(z^{i}\right)=v^{i}$. Clearly, $z^{1}+z^{2}=e$.

3. Any Pareto optimal allocation $a=\left(a^{1}, a^{2}\right)$ has the form $a^{1}=(1, \ldots, 1, \lambda, 0, \ldots, 0), a^{2}=$ $(0, \ldots, 0,1-\lambda, 1, \ldots, 1)$ with $a^{1}+a^{2}=e$.

4. $U^{i}\left(e-D^{3-i}\left(m, \bar{p}^{i}\right)\right)=M^{i}-m$ holds for $i \in I$ and $m \in\left[0, M^{i}\right]$.

5. For $m \in\left[0, M^{1}\right]$ we have $U^{1}\left(D^{1}\left(m, \bar{p}^{2}\right)\right)=h_{1}\left(1-\frac{m}{M^{2}}\right)$.

\section{Proof:}

1) First note that for a price system $p, D^{i}(m, p)$ is obtained as follows. Order the objects according to their utility/price ratio, i.e. $u_{j}^{i} / p_{j}$. Then agent $i$ first demands the object with the highest ratio, then the one with the second highest a.s.o. until his budget $m$ is used up. For $p=\bar{p}^{3-i}=u^{3-i}$ this means, we order the objects according to $u_{j}^{i} / u_{j}^{3-i}$, i.e. according to their substitution rates. With our assumptions that objects are already ordered in this way and different objects have different substitution rates, we conclude that $D^{1}\left(m, \bar{p}^{2}\right)$ consists of a unique vector of the form $(1, \ldots, 1, \lambda, 0, \ldots, 0)$ and $D^{2}\left(m, \bar{p}^{1}\right)$ is of the form $(0, \ldots, 0, \mu, 1, \ldots, 1)$ with $\lambda, \mu \in[0,1]$.

2) This immediately follows from our assumption that substitution rates are different across objects. Therefore, to any allocation $z$ of objects, the utility allocation of which is Pareto optimal in $V^{U}$, there does not exist a redistribution of $z$, so that every agent is equally well off.

3) Note that any Pareto efficient utility allocation $v \in V^{U}=\sum_{j \in N} V_{j}^{U}$ can be uniquely written as the sum of utility allocations in $V_{j}^{U}$, i.e. $v=\sum_{j} v_{j}$. All utility allocations $v_{j}$ as well as $v$ have to exhibit the same normal vector of a supporting hyperplane and, with our assumption on different substitution rates, it follows that for all but at most one $j$ we have either $v_{j}=\left(u_{j}^{1}, 0\right)$ or $v_{j}=\left(0, u_{j}^{2}\right)$. So, all but at most one object are completely allocated to some agent. With the assumption on the order of objects according to substitution rates, we conclude that the allocation of objects corresponding to $v$ takes the asserted form.

4) We prove the case $i=1$. Let $z^{2}:=D^{2}\left(m, \bar{p}^{1}\right)$. At price system $\bar{p}^{1}$ we have $u_{j}^{1} / \bar{p}_{j}^{1}=1$ for all $j \in N$. Hence, $D^{1}\left(m^{\prime}, \bar{p}^{1}\right)=\left\{x \in[0,1]^{n} \mid \bar{p}^{1} x=m^{\prime}\right\}$ collects all bundles, the worth of which under $\bar{p}^{1}$ is exactly $m^{\prime}$. Since $z^{2} \in B\left(m, \bar{p}^{1}\right)$ (and preferences are strictly monotonic), we know that $\bar{p}^{1} z^{2}=m$, hence $\bar{p}^{1}\left(e-z^{2}\right)=M^{1}-m$ and therefore $e-z^{2} \in D^{1}\left(M^{1}-m, \bar{p}^{1}\right)$. Note that $\left(e-z^{2}, z^{2}\right)$ is a Pareto efficient allocation, since agent 2 maximizes his utility on the budget set and agent $i$ can only be better off, when obtaining a higher income than $M^{1}-m{ }^{5}$ Therefore $U^{1}\left(e-z^{2}\right)=M^{1}-m$.

5) With part 4 , we obtain $U^{2}\left(e-D^{1}\left(m, \bar{p}^{2}\right)\right)=M^{2}-m=\left(1-\frac{m}{M^{2}}\right) M^{2}$ and since $\left(D^{1}\left(m, \bar{p}^{2}\right), e-\right.$ $\left.D^{1}\left(m, \bar{p}^{2}\right)\right)$ is Pareto efficient, we conclude $U^{1}\left(D^{1}\left(m, \bar{p}^{2}\right)\right)=h_{1}\left(1-\frac{m}{M^{2}}\right)$.

Note that as a consequence of part 1 of the lemma, we can conclude $D^{i}\left(m, \bar{p}^{3-i}\right) \geq$ $D^{i}\left(m^{\prime}, \bar{p}^{3-i}\right)$ (vector inequality), if $m \geq m^{\prime}$.

\footnotetext{
${ }^{5}$ In fact, the pair $\left(\bar{p}^{1} ;\left(e-z^{2}, z^{2}\right)\right)$ constitutes a Walrasian equilibrium of the underlying economy w.r.t. to the given income distribution. See Haake (2004) for further details.
} 


\section{Support Results}

Achieving a support result for a bargaining solution means executing the following task: One has to derive from each bargaining game $V^{U}$ a non-cooperative game $\Gamma^{U}$, so that payoffs in equilibrium coincide with the bargaining solution applied to the bargaining game at hand. The "rules" for deriving such a game should thereby not make use of knowledge about the underlying bargaining problem, but rather describe how $\Gamma$ is derived in general. Clearly, the "strength" of a support result is connected to the strength of the equilibrium concept that is used.

In this section, we will discuss two support results for the KS solution in the present context. First we describe rules for deriving a game in strategic form having exactly one Nash equilibrium. The equilibrium payoff coincides with the KS solution of the underlying bargaining game. Second, we derive a game in extensive form, which has multiple subgame perfect equilibria. However, there are no coordination problems involved, as all equilibria have s the same payoff; again the KS solution.

Payoffs in both games, respectively the final allocations of objects, are achieved by endowing agents with a specific amount of money and letting them demand objects at prices $\bar{p}^{1}$ or $\bar{p}^{2}$.

\subsection{Unique Nash equilibrium support}

For $U \in \mathcal{U}$ we construct a game $\Gamma^{U}=\left(S^{1}, S^{2}, F^{1}, F^{2}\right)$ as follows: Strategy spaces are $S^{1}=S^{2}=[0,1]$. For any pair of strategies $\gamma=\left(\gamma^{1}, \gamma^{2}\right) \in S^{1} \times S^{2}$, we first determine an allocation $g(\gamma)=\left(g^{1}(\gamma), g^{2}(\gamma)\right) \in \mathcal{A}$ of objects as follows:

(g1) Determine for $i \in I$ agent $i$ 's demand at income level $\left(1-\gamma^{3-i}\right) \cdot M^{3-i}$, at prices $\bar{p}^{3-i}=u^{3-1}$, i.e. determine $x^{i}(\gamma):=D^{i}\left(\left(1-\gamma^{3-i}\right) \cdot M^{3-i}, \bar{p}^{3-i}\right)$.

Set $z^{i}(\gamma):=e-x^{3-i}(\gamma)$. If $z^{1}(\gamma)+z^{2}(\gamma) \leq e$ holds, then define $g(\gamma):=\left(z^{1}(\gamma), z^{2}(\gamma)\right)$.

(g2) Otherwise $\left(z^{1}(\gamma)+z^{2}(\gamma) \not \leq e\right)$, determine $\hat{z}^{i}(\gamma):=D^{i}\left(\left(1-\gamma^{i}\right) \cdot M^{3-i}, \bar{p}^{3-i}\right)$ and set

$$
g^{i}(\gamma):=\left\{\begin{array}{lll}
z^{i}(\gamma) & , \text { if } & z^{i}(\gamma)+\left(e-\hat{z}^{i}(\gamma)\right) \leq e \\
\hat{z}^{i}(\gamma), & \text { otherwise }
\end{array}\right.
$$

Payoffs in $\Gamma^{U}$ are given by evaluation of the resulting allocation, i.e. $F^{i}(\gamma)=U^{i}\left(g^{i}(\gamma)\right)(i \in I)$.

Theorem 1. For each $U \in \mathcal{U}$ the game $\Gamma^{U}$ has a unique Nash equilibrium $\bar{\gamma}$. This is given by $\bar{\gamma}^{1}=\bar{\gamma}^{2}=\tau\left(V^{U}\right)$. Furthermore, $F(\bar{\gamma})=K\left(V^{U}\right)$.

Proof: Fix $U \in \mathcal{U}$ and set $\tau:=\tau\left(V^{U}\right)$, hence we examine $\bar{\gamma}=(\tau, \tau)$.

Step 1: Note first, that $g(\bar{\gamma})$ is determined according to $(g 1)$. With part 4 of Lemma 1, we know $U^{i}\left(z^{i}(\bar{\gamma})\right)=U^{i}\left(e-D^{3-i}\left((1-\tau) M^{3-i}, \bar{p}^{3-i}\right)\right)=\tau M^{i}(i=1,2)$ and hence $\left(U^{1}\left(z^{1}(\bar{\gamma})\right), U^{2}\left(e-z^{1}(\bar{\gamma})\right)\right)=K\left(V^{U}\right)=\left(U^{1}\left(e-z^{2}(\bar{\gamma})\right), U^{2}\left(z^{2}(\bar{\gamma})\right)\right)$, which implies $z^{1}(\bar{\gamma})=$ $e-z^{2}(\bar{\gamma})$. Therefore $g(\bar{\gamma})=\left(z^{1}(\bar{\gamma}), z^{2}(\bar{\gamma})\right)$ and $F^{i}\left(g^{i}(\bar{\gamma})\right)=K^{i}\left(V^{U}\right)(i \in I)$.

Step 2: Next, we show that $\bar{\gamma}$ is a Nash equilibrium in $\Gamma^{U}$. Suppose agent 1 deviates to $\sigma^{1}<\tau$. Since $e-D^{2}\left((1-\tau) \cdot M^{1}, \bar{p}^{1}\right) \geq e-D^{2}\left(\left(1-\sigma^{1}\right) \cdot M^{1}, \bar{p}^{1}\right)$ holds ${ }^{6}$, the function $g$ is still determined according to $(g 1)$ and hence $F^{1}\left(\sigma^{1}, \tau\right)=U^{1}\left(g^{1}\left(\sigma^{1}, \tau\right)\right) \leq U^{1}\left(g^{1}(\tau, \tau)\right)=F^{1}\left(\sigma^{1}, \tau\right)$.

\footnotetext{
${ }^{6}$ Use part 1 of Lemma 1 and the fact that demand is increasing in income.
} 
If agent 1 deviates to $\rho^{1}>\tau$, we conclude that $g$ is determined by $(g 2)$. Suppose $z^{1}\left(\rho^{1}, \tau\right)+\left(e-\hat{z}^{1}\left(\rho^{1}, \tau\right)\right) \leq e$ were true. Then $\left(z^{1}\left(\rho^{1}, \tau\right), e-\hat{z}^{1}\left(\rho^{1}, \tau\right)\right) \in \mathcal{A}$ is a feasible allocation. Using Lemma 1 we compute its utility allocation $\left(U^{1}\left(z^{1}\left(\rho^{1}, \tau\right)\right), U^{2}\left(e-\hat{z}^{1}\left(\rho^{1}, \tau\right)\right)\right)=$ $\left(\rho^{1} M^{1}, \tau M^{2}\right)>\left(\tau M^{1}, \tau M^{2}\right)=K\left(V^{U}\right)$, which contradicts Pareto efficiency of the KS solution. It follows again with part 5 of Lemma 1 that $F^{1}\left(\rho^{1}, \tau\right)=U^{1}\left(\hat{z}^{1}\left(\rho^{1}, \tau\right)\right)=h_{1}^{U}\left(\rho^{1}\right)<$ $h_{1}^{U}(\tau)=\tau M^{1}=F^{1}(\bar{\gamma})$ has to hold. Analogous arguments apply for agent 2 .

Step 3: Step 2 shows that agent $i$ can assure himself a payoff of $\tau M^{i}$ by choosing $\bar{\gamma}^{i}=\tau$. Therefore, the payoff in any other equilibrium has to be at least this amount for both agents. But the only utility allocation in $V^{U}$ that does satisfy this condition is $K\left(V^{U}\right)$. It is then immediate that $\bar{\gamma}$ is the only strategy profile with payoff $K\left(V^{U}\right)$ and therefore the only Nash equilibrium in $\Gamma^{U}$.

\subsection{Subgame Perfect support}

Next, we derive an extensive form game $\Sigma^{U}$ from a bargaining game $V^{U} \in \mathcal{V}$. Again, we first obtain an allocation as the result of strategy choices. The rules are as follows:

Stage 1 Agent 1 selects $\eta \in[0,1]$.

Stage 2 Agent 2 is entitled to either choose a bundle $z^{2}=z^{2}(\eta) \in B\left((1-\eta) M^{1}, \bar{p}^{1}\right)$ or to pass to the next stage. In the former case, the final allocation is $\left(e-z^{2}, z^{2}\right) \in \mathcal{A}$.

Stage 3 Agent 1 is entitled to choose a bundle $z^{1}=z^{1}(\eta) \in B\left((1-\eta) M^{2}, \bar{p}^{2}\right)$. The final allocation is $\left(z^{1}, e-z^{1}\right) \in \mathcal{A}$.

Again, payoffs in $\Sigma^{U}$ are determined by evaluation of the final allocation with $U^{i}(\cdot)$.

Theorem 2. Let $\bar{z}=\left(\bar{z}^{1}, \bar{z}^{2}\right)$ be the final allocation and $\bar{\eta}$ be the chosen parameter at Stage 1 in a subgame-perfect equilibrium of $\Sigma^{U}$. Then we have $\bar{\eta}=\tau\left(V^{U}\right)$ and $\left(U^{1}\left(\bar{z}^{1}\right), U^{2}\left(\bar{z}^{2}\right)\right)=$ $K\left(V^{U}\right)$.

\section{Proof:}

First, in any subgame perfect equilibrium, if either agent 2 or 1 chooses a bundle from the budget set (at stage 2 or 3 ), he will choose $z^{2}=D^{2}\left((1-\eta) M^{1}, \bar{p}^{1}\right)$ or $z^{1}=D^{1}\left((1-\eta) M^{2}, \bar{p}^{2}\right)$, respectively. By parts 4 and 5 of Lemma 1 , we know that $U^{1}\left(e-z^{2}\right)=\eta \cdot M^{1}$ and $U^{2}\left(e-z^{1}\right)=$ $\eta \cdot M^{2}$, which means $U^{1}\left(z^{1}\right)=h_{1}^{U}(\eta)$.

At stage 2, agent 2 compares his payoff from choosing $z^{2}$ himself with $U^{2}\left(e-z^{1}\right)$, which he anticipates when passing to the next round. Since the respective allocations $\left(e-z^{2}, z^{2}\right)$ and $\left(z^{1}, e-z^{1}\right)$ are Pareto efficient, we have that $U^{2}\left(z^{2}\right)>U^{2}\left(e-z^{1}\right)$, if and only if $U^{1}\left(e-z^{2}\right)<U^{1}\left(z^{1}\right)$. so agent 2 takes his decision as to minimize agent 1 's payoff.

Therefore, at stage 1, agent 1 faces a payoff of $\min \left(U^{1}\left(z^{1}\right), U^{1}\left(e-z^{2}\right)\right)=\min \left(h_{1}^{U}(\eta), \eta M^{1}\right)$. To maximize this expression, agent 1 chooses $\bar{\eta}$ to equate $h_{1}^{U}(\bar{\eta})=\bar{\eta} M^{1} \cdot{ }^{7}$ Hence, $h^{U}(\bar{\eta})=$ $\left(\bar{\eta} M^{1}, \bar{\eta} M^{2}\right)$, so $\bar{\eta}=\tau\left(V^{U}\right)$ and the equilibrium payoff coincides with $K\left(V^{U}\right)$.

Note that in equilibrium agent 2 is indifferent between choosing himself or letting agent 1 choose at stage 3 . Nonetheless, there is no coordination problem at all, since with the unique equilibrium parameter $\bar{\eta}=\tau\left(V^{U}\right)$ the resulting allocations yield the same payoffs. With our assumption on different substitution rates, agent 1 and 2 will even choose the same allocation of objects.

\footnotetext{
${ }^{7}$ Recall that $h_{1}^{U}(\eta)$ is decreasing in $\eta$.
} 


\section{Discussion and Further Results}

Although the game $\Gamma^{U}$ is a one-shot game, we may think of payoff functions as to be determined in several steps. First, according to strategy choice $\gamma^{i} \in[0,1]$ of agent $i$, agent $3-i$ is equipped with an income of $\left(1-\gamma^{i}\right) M^{3-i}$, thus with a $1-\gamma^{i}$ share of the value of the total object set under prices $\bar{p}^{3-i}$. Next, agent $3-i$ selects a bundle w.r.t. his income and leaves the remaining objects to agent $i$ (the bundle $z^{i}(\gamma)$ in $(g 1)$ ). Assuming that $3-i$ chose the utility maximizing bundle, we have $U^{i}\left(z^{i}(\gamma)\right)=\gamma^{i} M^{i}$. But this will be the final payoff, only if the two bundles $z^{1}(\gamma), z^{2}(\gamma)$ constitute a feasible allocation. In this sense, we check compatibility of $\gamma^{1}$ and $\gamma^{2}$. Intuitively, on the one hand a "higher" $\gamma^{i}$ proposes a higher utility, but on the other hand makes it less likely that the we have a feasible allocation in $(g 1)$. Now, what happens, if $\left(z^{1}(\gamma), z^{2}(\gamma)\right)$ fails to be an allocation? Then we examine each $\gamma^{i}$ once again. Now agent $i$ himself will be endowed with a $\left(1-\gamma^{i}\right)$ share of $M^{3-i}$ and is supposed to choose a utility maximizing bundle (this is $\left.\hat{z}^{i}(\gamma)\right)$. Observe that $U^{3-i}\left(e-\hat{z}^{i}(\gamma)\right)=\gamma^{i} M^{3-i}$. According to $(g 2)$, agent $i$ still obtains $z^{1}(\gamma)$, only if it is possible to provide a $\gamma^{i}$ share of total utilities to both agents (in that case $\left(z^{1}(\gamma), e-\hat{z}^{1}(\gamma)\right)$ is feasible). But, if $\gamma^{i}$ is so "high" that this would not be possible, then agent $i$ gets away with $\hat{z}^{i}(\gamma)$, i.e., the bundle chosen w.r.t. a "low" income.

Whereas we assumed utility maximizing choice in $\Gamma^{U}$, we let agents freely choose in $\Sigma^{U}$. But, since the bundle chosen by either agent 2 or 1 is the final bundle for that agent, utility maximizing prevails in any subgame perfect equilibrium. Inspecting the decision to be taken by agent 2 at stage 2 reveals that he has to decide between two (Pareto efficient) allocations. Suppose, $\eta$ was chosen at stage 1 . Then either agent 1 obtains an $\eta$ share of his maximal utility (which is the case, if agent 2 chooses himself), or agent 2 passes and obtains $\eta M^{2}$ (agent 1 chooses at stage 3 ). As in $\Gamma^{U}$ a "high" $\eta$ can backfire for agent 1 , as agent 2 may pass to stage 3 , where agent 1 is endowed with a "low" income of $(1-\eta) M^{2}$. Conversely, a "low" $\eta$ gives incentives for agent 2 to choose himself at stage 2 with a "high" income. In effect, there remains a "max-min problem" for agent 1 as this is also incorporated in the definition of the KS solution. This kind of tradeoff was also observed by Trockel (2000) in a welfaristic context.

We should add a note on the role of prices. Although one can formally show that the two price systems $\bar{p}^{i}(i \in I)$ constitute Walrasian equilibrium prices ${ }^{8}$, we shall not regard them as competitive prices. Put in other words, these prices shall not be viewed as being formed under perfect competition, but result from application of the (normative) solution concept of Walrasian equilibrium. Therefore, we rather use equilibrium prices as an instrument to achieve certain allocations. So, price taking is not understood as a consequence of perfect competition but belongs to the rules of the game.

Both games $\Gamma^{U}$ and $\Sigma^{U}$ satisfy a property that was termed full range property by Trockel (2000). This means that the rules of the game allow for any feasible allocation in $\mathcal{A}$ as the outcome of the game, or, put in other words, any utility allocation $V^{U}$ is attainable as payoff from strategic interaction. So, by playing the game instead of signing an agreement, the agents do not lose any of their allocation possibilities. For example, in $\Gamma^{U}$ a high $\gamma^{1}$ can be compatible with a "low" $\gamma^{2}$, so that a high payoff for agent 1 is (in principle) possible.

Observe that the assumption of ordered and mutually different substitution rates does not

\footnotetext{
${ }^{8}$ see Haake (2004) for details.
} 
constitute a material restriction. In case there are different objects having the same substitution rate, we lose the one-to-one correspondence between Pareto efficient utility and object allocations. Moreover, the demand sets in part 1 of Lemma 1 are no longer singletons. However, we will always be able to select an appropriate allocation, the utility allocation of which satisfies required properties.

The reader may dislike that there are two different price systems involved to obtain payoffs. As Haake (2004) shows the price system $\bar{p}$ with $\bar{p}_{j}=\left(u_{j}^{1} \cdot u_{j}^{2}\right)^{1 / 2}$ is always an equilibrium price system and therefore individual demand leads to an efficient allocation. As it is basically shown there, we get support results for the superadditive bargaining solution introduced by Perles \& Maschler (1981). If one replaces $\bar{p}^{i}$ by $\bar{p}$ in $\Gamma^{U}\left(\Sigma^{U}\right)$, the results go through with $K\left(V^{U}\right)$ replaced by the PM solution. The equilibrium strategies in $\Gamma^{U}$ are given by $\bar{\gamma}=(1 / 2,1 / 2)$ as well as $\bar{\eta}=1 / 2$ in $\Sigma^{U}$.

\section{References}

Bergin, J. \& J. Duggan (1999): "An Implementation-Theoretic Approach to noncooperative Foundations", Journal of Economic Theory, 86, 50-76.

Dagan, N. \& R. Serrano (1998): "Invariance and Randomness in the Nash Program for coalitional Games", Economics Letters, 58, 43-59.

HaAke, C.-J. (2000): "Supporting the Kalai-Smorodinsky Bargaining Solution", Institute of Mathematical Economics, Bielefeld University, Working Paper No. 301 (rev. version).

(2004): "Dividing by Demanding: Object Division through Market Procedures", Institute of Mathematical Economics, Bielefeld University, Working Paper No. 359.

Kalai, E. \& M. Smorodinsky (1975): "Other Solutions to Nash's Bargaining Problem", Econometrica, 43, 513-518.

MiYagaWA, E. (2002): "Subgame-perfect implementation of bargaining solutions", Games and Economic Behavior, 41(2), 292-308.

Moulin, H. (1984): "Implementing the Kalai-Smorodinsky Bargaining Solution", Journal of Economic Theory, 33, 32-45.

Perles, M. \& M. Maschler (1981): "The Super-Additive Solution for the Nash Bargaining Game", International Journal of Game Theory, 10(3/4), 163-193.

Serrano, R. (1997): "A Comment on the Nash Program and the Theory of Implementation", Economics Letters, 55, 203-208.

Trockel, W. (1999): "Unique Nash Implementations for a Class of Bargaining Solutions", International Game Theory Review, 1, 267-272.

(2000): "Implementations of the Nash Solution Based on its Walrasian Characterizations", Economic Theory, 16, 277-294.

(2002): "Integrating the Nash Program into Mechanism Theory", Review of Economic Design, 7(1), 27-43. 